\title{
Adaptive Microcalcification Detection in Computer Aided Diagnosis
}

\author{
Ho-Kyung Kang ${ }^{1}$, Sung-Min Kim², Nguyen N. Thanh ${ }^{1}$, \\ Yong Man Ro ${ }^{1}$, and Won-Ha Kim ${ }^{3}$ \\ ${ }^{1}$ Multimedia group, Information and Communication University, Korea \\ \{kyoung, nnthanh, yro\}@icu.ac.kr \\ ${ }^{2}$ Department of Biomedical Engineering, School of Medicine, KonKuk University, Korea \\ smkim@kku.ac.kr, \\ ${ }^{3}$ Kyung Hee University, Korea \\ wonha@khu.ac.kr
}

\begin{abstract}
Microcalcification detection is an important part of early breast cancer detection. In this paper, we propose a microcalcification detection method in mammography CAD (computer-aided diagnosis) system. The proposed microcalcification detection includes two parts. One is adaptive mammogram enhancement algorithm using homomorphic filtering in wavelet. The filter parameters are determined by background characteristics. The other is multi-stage microcalcification detection method. To verify our algorithm, we performed experiments and measured free-response operation characteristics (FROC) curve. The results show that the proposed microcalcification detection method is more robust for fluctuating noisy environments.
\end{abstract}

\section{Introduction}

From recent medical report, breast cancer is a leading cause of death for middle-aged women in USA and Europe. Thus, early detection and improvement in cancer treatments can reduce the mortality of breast cancer. Especially, the detection of microcalcification is a major part of diagnosis in early stage breast cancer. However, the microcalcification is too small to detect by palpable breast diagnosis. It is known that mammogram is the best modality to detect microcalcification [1].

Various types, sizes, and distributions of microcalcification make it difficult to detect actual microcalcification. In NMD (ACR National Mammography Database), 11 types of microcalcification and 5 types of distribution are categorized. These types of calcification are classified as benign calcification that is usually larger than the calcification associated with malignancy. Regional distributed calcifications scattered in large volume are not malignant. But the clustered calcifications are very dangerous type of microcalcification [2].

Image resolution and film contrast of mammogram are major problems in mammography. Numerous technologies and equipments have been developed during last several

\footnotetext{
* Images were provided by University of South Florida DDSM (Digital Database for Screening Mammography). This paper is supported by the development of digital CAD system (02-PJ3PG6-EV06-0002) of Ministry of Health and welfare in Republic of Korea.
} 
years. However, mammography has remained as one of the diagnostic modalities which are being enhanced. In mammogram, high attenuation properties and small size of microcalcification are major reasons of poor visualization and small lesions. To improve the visibility of breast cancer, image enhancement methods have been performed $[3,4,5,6,7,8]$. The aim of these studies was to increase the contrast of microcalcification.

These previous algorithms may effectively detect microcalcifications. But some mammograms are taken from different environment such as different noise condition, $\mathrm{X}$-ray intensity and concentration of sensitizer of mammogram films. In most previous works, they took the same parameters of image enhancement, denoising, and feature extraction. Some of DDSM (Digital Database for Screening Mammography) images include various kinds of noise in background and the noise is diffused through the breast area. Therefore, we must eliminate these kinds of noise to improve the performance of microcalcification detection.

In this paper, we propose an adaptive microcalcification detection method, which uses an adaptive image enhancement and noise reduction by using noise characteristics in background region of each mammogram. This paper consists of several sections. In Sect. 2, we describe our microcalcification detection system. Section 3 presents the performance of our method in DDSM database. We make conclusions in Sect. 4.

\section{Adaptive Microcalcification Detection}

The entire scheme of the proposed method is shown in Fig.1. Total system is composed into major two parts. One is image enhancement and the other is detection using ANN (Artificial Neural Network).

\begin{tabular}{c}
$\begin{array}{c}\text { Mammogram } \\
\text { Image }\end{array}$ \\
\hline $\begin{array}{c}\text { Calcification } \\
\text { Marking }\end{array}$
\end{tabular}

Fig. 1. Total system of our proposed method

In image enhancement part, we take background areas to obtain noise characteristics. Next, we use adaptive homomorphic enhancement and noise reduction in breast areas. The enhancement parameters are determined by noise characteristics of background. In detection part, first we get pixel information to find a potential microcalcification in breast areas that is to find ROI (Region of Interest). In microcalcification detection, entire image is decomposed into sub images corresponding to ROIs and microcalcifications are detected within these sub images. 


\subsection{Adaptive Mammogram Enhancement Using Homomorphic Filtering}

The homomorphic filter function decreases the energy of low frequencies and increases those of high frequencies in the image. The homomorphic filter is used to find the gain $K_{m}$ [4]. With the mammography images, the homomorphic filter gives contrast stretching for lower gray level, thereby enhancing the contrast. Based on the characteristics of homomorphic filter function, we determined the gain of mapping function, i.e., weighting wavelet coefficients of channels corresponding to homomorphic filter function. Figure 2 represents the gain $K_{m}$ that is determined according to the discrete homomorphic filtering.

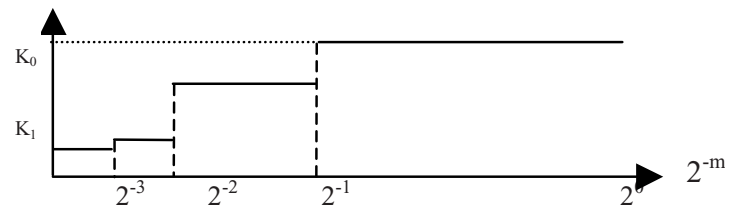

Fig. 2. Filter function for applying to wavelet coefficients

Figure 3 is a diagram of homomorphic filtering approach in this framework. Here, we first take logarithmic function for input signal. It also inverts the exponential operation caused by the radioactive absorption, which is generated in the process of obtaining mammography image.

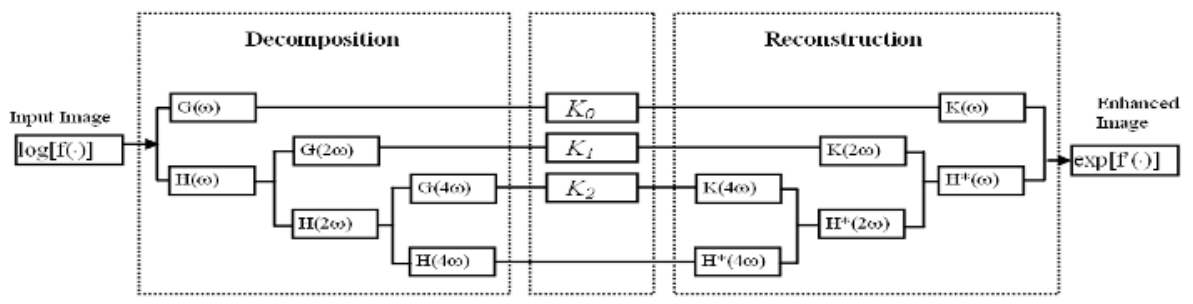

Fig. 3. Homomorphic filtering approach for image enhancement

Noise reduction is a considerable issue in image enhancement. One method of denoising is wavelet shrinkage that was presented in [3]. We can see that each mammogram contains its own noise characteristics because mammograms are taken from different environment. So applying the same parameters in wavelet shrinkage for every mammogram is not efficient. Taking into account noise properties of each mammogram, we propose an adaptive method for mammogram enhancement.

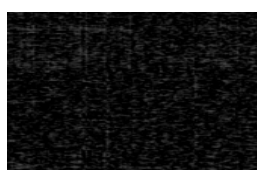

(a)

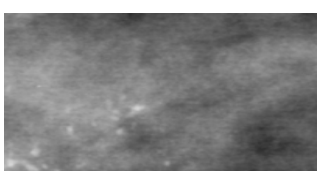

(b)

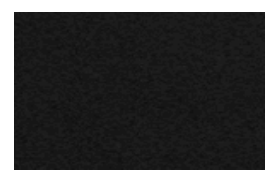

(c)

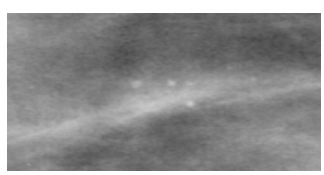

(d)

Fig. 4. Examples of background noises and microcalcifications. (a),(b) are high noise (var.>100) in background and breast, (c),(d) are low noise (var.<50) in background and breast 
Figure 4 (a), (b) images have some colored noise with high variance value. One can notice noise effect in breast area. To obtain noise characteristics of the image, the background of mammogram is segmented by thresholding the value combining the gray-level, mean and variance of pixels. The background areas are supposed to contain the noise of image. So we can take noise characteristic in this area. The noise characteristic is measured by background noise variance, which can be written as

$$
\operatorname{var}_{\text {background }}=\frac{1}{N_{\text {background }}} \sum_{(x, y) \text { background }}\left\{(I(x, y)-\text { mean }(x, y))^{2}\right\},
$$

where $N_{\text {background }}$ is the number of pixel in background area. If high variance of background noise exists, we need to reduce the gain of homomorphic filter in high frequency domain as

$$
K_{m}{ }^{\prime}=K_{m} \times \frac{A}{\operatorname{var}_{\text {background }}+A}, \quad \text { if } m=0 \text { and } m=1
$$

where $A$ is constant value to normalize noise variance. The $m$ is level of wavelet, $K_{m}$ means the gains of each wavelet level. In (2), $m=0$ means highest frequency level in wavelet and $m=1$ means second highest wavelet level.

Further, an adaptive method of denoising is included in the enhancement framework in wavelet domain. To achieve the edge-preserved denoising, nonlinear wavelet shrinkage method is proposed. A shrinking operator $(S(u))$ for denoising can be written as

$$
S(x)=\left\{\begin{array}{l}
\operatorname{sign}(u) \times\left(|u|-\operatorname{var}_{\text {backgrount }}{ }^{2} / \sigma\right) \text { if }|u|>\operatorname{var}_{\text {backgrount }}{ }^{2} / \sigma \\
0 \quad \text { otherwise, }
\end{array}\right.
$$

where $\sigma$ is variance of reconstructed image using wavelet coefficients in a sub-band. $\operatorname{sign}(u)$ means positive or negative sign of wavelet coefficient $u$. Threshold in this wavelet shrinkage is called a nearly optimal threshold [9].

Taking adaptive gains of high frequency area of wavelet domain and optimal denoising operators, microcalcification can be enhanced and also noise can be enhanced in breast area.

\subsection{Microcalcification Detection}

After adaptive image enhancement, we extract features to find ROIs which contain potential microcalcifications. Further, an artificial neural network with two hidden layers is employed to detect the pixels that may belong to microcalcifications. The features are obtained using statistical characteristics of gray level. We used two effective features: median contrast at position and contrast to noise ratio same as in [8].

In the neural network, inputs are two features mentioned above. And outputs are two categories of pixels, e.g., the pixel belonging to the ROI or not belonging to. In this paper, the first layer of ANN has 10 units and second layer has 4 units.

To train the neural network, fifty mammogram images containing cancer are selected. Pixels in calcification area of these images are obtained to make positive training samples. Negative training samples are obtained randomly in the mammogram 
images of normal cases. Using the trained ANN, potential microcalcifications are found in a mammogram image to be tested. Then, the close detected pixels are grouped into one ROI as potential microcalcification.

From each potential microcalcification, which is found in the ROI detection, we find a sub-image whose size are triples of the ROI and its center is located at the center point of the ROI. In the sub-image, some features are obtained using local edge histogram, high-pass mask filter and Number of neighboring ROI.

In the local edge histogram, we measured two features with edge direction using $2 \times 2$ edge mask shown in Fig. 5. One is the maximum value of one direction and the other is non-directional edge. Using the local edge histogram, we can obtain high nondirectional edge histogram value in microcalcification region while, in tissue or the mammary gland case, directional edges are emphasized. In the ROI detection, we found suspicious pixels such as microcalcification and abrupt change area. Using this edge information, above ambiguous pixels in breast area could be differentiated.

\begin{tabular}{|l|l|}
\hline 1 & -1 \\
\hline 1 & -1 \\
\hline
\end{tabular}

vertical edge

\begin{tabular}{|c|c|}
\hline 1 & 1 \\
\hline-1 & -1 \\
\hline
\end{tabular}

horizontal edge

\begin{tabular}{|c|c|}
\hline$\sqrt{2}$ & 0 \\
\hline 0 & $-\sqrt{2}$ \\
\hline
\end{tabular}

Diagonal edge $45^{\circ}$

\begin{tabular}{|c|c|}
\hline 0 & $\sqrt{2}$ \\
\hline$-\sqrt{2}$ & 0 \\
\hline
\end{tabular}

Diagonal edge $135^{\circ}$

\begin{tabular}{|c|c|}
\hline 2 & -2 \\
\hline-2 & 2 \\
\hline
\end{tabular}

Nondirectional edge

Fig. 5. Directional filter for edge detection

The two features in local edge histogram can be written as

$$
\begin{gathered}
\text { Feature_Edge } e_{\text {directional }}=\max \left(E_{\text {verticaledge }}, E_{\text {horizontaledge }}, E_{\text {diagonaledge } 45^{\circ}}, E_{\text {diagonaledgel } 35^{\circ}}\right), \\
\text { Feature_Edge } e_{\text {non-directional }}=E_{\text {nondirectionaledge }},
\end{gathered}
$$

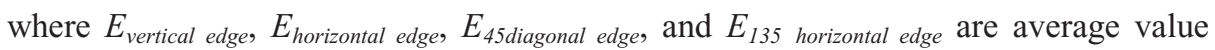
after filtering shown in Fig.5, which represent four kinds of directional edge information.

To obtain the feature using high pass mask filter, the feature for the high pass mask can be obtained by

$$
\text { Feature_Highpass }=9 \times \sum_{(i, j) \in R O I} p(i, j)-\sum_{(i, j) \in W} p(i, j),
$$

where, $W$ represents total window of high pass mask and ROI means potential microcalcification. For this feature, microcalcification is supposed to has higher value compared with adjacent area.

Finally the feature using number of neighboring ROI can be obtained by

$$
\text { Feature } \_N_{\text {ROII }}\left(x_{1}, y_{1}\right)=\sum R O I\left(x_{i}, y_{i}\right) \text { if }\left(x_{1}-x_{i}\right)^{2}+\left(y_{1}-y_{i}\right)^{2}<R^{2},
$$

where, $R O I(x, y)$ means center position of an ROI. $R$ is threshold which is related with microcalcification distribution. Equation (7) represents how many potential microcalcifications are located in adjacent area. To detect malignant microcalcification, the 
distribution type of microcalcification is important. Real microcalcification is distributed in a way of highly clustering with neighboring potential microcalcification. Therefore, we believe high value of the feature means real microcalcification.

A back-propagation neural network is used to detect the microcalcification with features mentioned in above. The marked calcification areas of mammogram, which are confirmed by medical doctors, are used as positive training data. ROI in the normal areas are used as negative training data.

\section{Experiment}

To verify the effectiveness of the proposed method, experiments are performed with DDSM mammogram database. In DDSM database, the pixel size of microcalcifications ranges from $1 \times 1$ to $10 \times 10$, resolution of mammogram image is $50 \mu \mathrm{m} / \mathrm{pixel}$, and gray level depths are 12bit and 16bit. The ANN training data and test data are obtained from DDSM database. The training database contains 56 cases of microcalcification and 56 cases of normal. And test data contains 118 cases of microcalcification and 118 cases of normal. Figure 6 (a) shows an original image, (b) is a local histogram enhanced image, (c) is a homomorphic enhancement image and (d) is adaptive enhancement image using the proposed algorithm. In Fig. 6 (b), (c) and (d), one can see more pattern of microcalcification than original image. Histogram stretched image is known to give good detail information of microcalcifications [3].

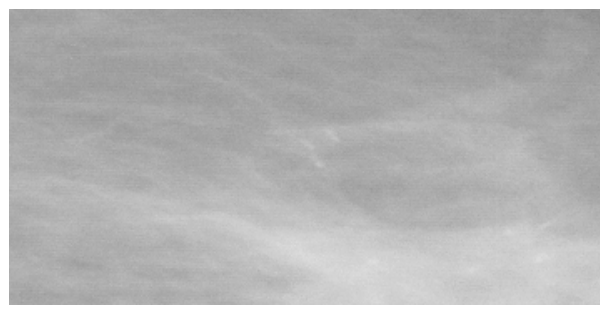

(a) Original image

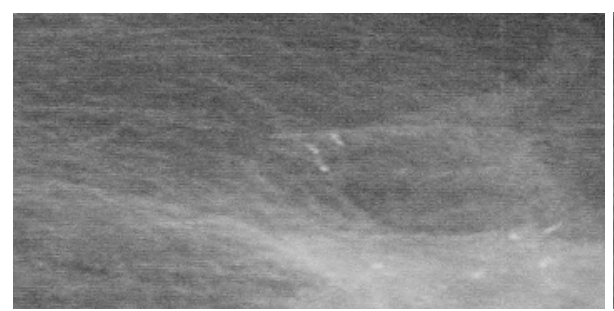

(c) Homomorphic filtering image

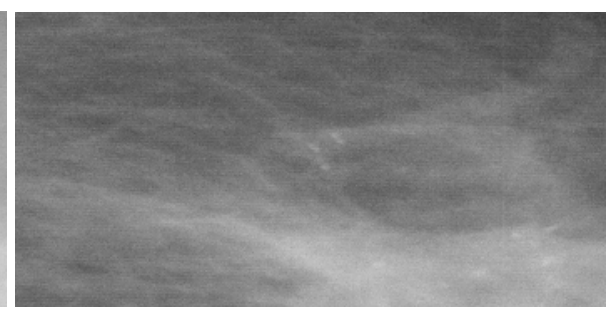

(b) Histogram stretched image

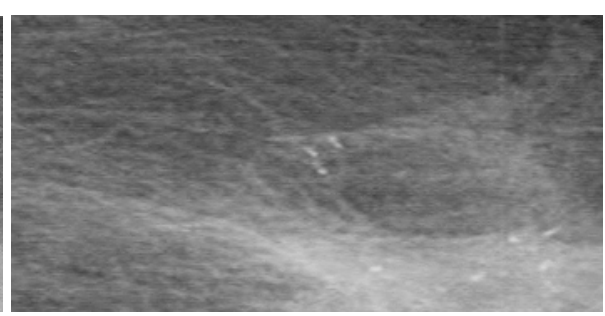

(d) Proposed method

Fig. 6. Examples of enhancement images

A quantitative measure of contrast improvement is calculated using contrast improvement index (CII) [3]. It is defined by the ratio of enhanced contrast and original 
contrast. Table 1 shows CII for original image and homomorphic enhanced image and adaptive enhanced in this paper. In Table 1, C means contrast value of an object, and standard deviation (STD.) of noise is obtained by background noise. As is seen, the proposed method gives higher CII value while smaller noise. Therefore proposed algorithm reduces noise compare with homomorphic filter.

Table 1. One example of Contrast Improvement Index and noise (Low noise case)

\begin{tabular}{|c|c|c|c|}
\hline & Original Image & Homomorphic filtering & Proposed Method \\
\hline C & 0.0183 & 0.0600 & 0.0710 \\
\hline CII & - & 3.2860 & 3.8858 \\
\hline STD. of noise & 23.1 & 11.5 & 10.3 \\
\hline
\end{tabular}

Because of the low contrast, original image has relatively low performance in microcalcification detection. We compared microcalcification detection using the proposed method with other methods such as histogram stretching and homomorphic enhancement method.

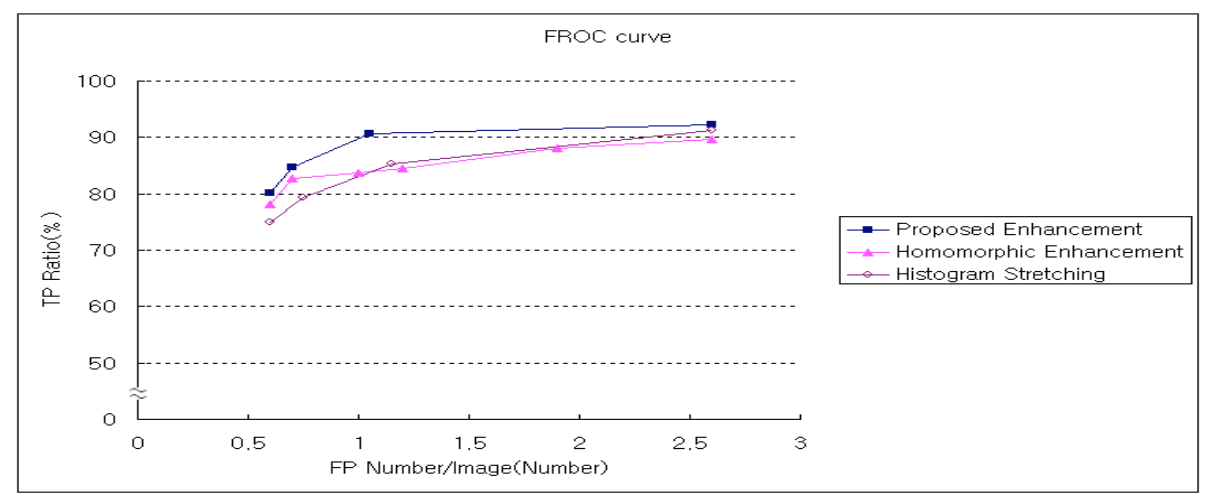

Fig. 7. ROC curve of microcalcification detection

To evaluate the performance of microcalcification detection, we measured FROC (free-response operation characteristics) curve shown in Fig.7. In FROC curve, we test 3 kinds of image enhancement methods. One is proposed method. Others are histogram stretching method and homomorphic enhancement method. TP ratio means true positive detection ratio, which shows how many microcalcifications are detected. And FP number/image means false positive number.

In the FROC curve, the proposed method shows higher TP ratio value than others methods at same FP number. The result of histogram stretching TP ratio is $79.3 \%$ for $0.75 \mathrm{FP}$ number/image. In homomorphic case, TP ratio is $82.8 \%$ for $0.7 \mathrm{FP}$ number/image. In proposed method, TP ratio is $84.6 \%$ for $0.7 \mathrm{FP}$ number/image. In Fig. 7 , the proposed method show better result for every FP value. Homomorphic enhancement case is almost same as with proposed method for low FP number. But, this method could amplify noises which could be dealt with similar feature as microcalcification. Therefore it could have poor performance for high FP number/image. 


\section{Conclusion}

Mammogram contains fluctuating noise characteristics because mammograms are taken from different environment, such as different noise condition, X-ray intensities and concentration of sensitizer of mammogram films. Therefore, the same parameters in enhancement gain and denoising for every mammogram are not efficient.

In this paper, we proposed an algorithm where noise characteristic is estimated in background area and eliminated in breast area. Therefore, we can obtain more enhanced mammogram using modified homomorphic filtering and adaptive denoising methods. Experimental result shows that the proposed algorithm gives better result compared with conventional image enhancement methods.

\section{References}

1. Johns, P. C., Yaffe, M. J.: X-ray characterization of normal and neoplastic breast tissues. Physics in Medicine and Biology. 32(6) (1987) 675-695

2. ACR National Mammography Database(NMD) http://www.acr.org/

3. Laine, A., Fan, J., Yang, W.: Wavelets for contrast enhancement of digital mammography. IEEE Engineering in Medicine and Biology Magazine 145 (1995) 536-550

4. Yoon, J. H., Ro, Y. M.: Enhancement of the contrast in mammographic images using the homomorphic filter method. IEICE Transactions on Information and Systems 85-D(1) (2002) 291-297

5. Karssemeijer, N.: A stochastic model for automated detection of calcifications in digital mammograms. Proc. 12th Int. Conf. Information Processing Medical Imaging (1991) 227238

6. Strickland, R. N., Hahn, H. I.: Wavelet transform for detecting microcalcifications in mammograms. IEEE Trans. Med. Imag. 15 (1996) 218-229

7. Yu, S., Guan, L.: A CAD system for the automatic detection of clustered microcalcifications in digitized mammogram film. IEEE Trans. Med. Imag. 19(2) (1998) 115-126

8. Sakellaropoulos, P., Costaridou, L., Panayiotakis, G.: A wavelet-based spatially adaptive method for mammographic contrast enhancement. Physics in Medicine and Biology 48 (2003) 787-803

9. Chang, S. G., Yu, B., Vetterli, M.: Adaptive wavelet thresholding for image denoising and compression. IEEE Trans. Image Processing 9(9) (2000) 1532-1546

10. Zheng, B., Qian, W., Clarke, L. P.: Digital mammography: Mixed feature neural net-work with spectral entropy decision for detection of microcalcifications. IEEE Trans. Med. Imag. 15 (1996) 589-597

11. Yoshida, H., Zhang, W., Cai, W., Doi, K., Nishikawa, R. M., Giger, M. L.: Optimizing wavelet transform based on supervised learning for detection of microcalcifications in digi-tal mammograms. Proc. IEEE ICIP 3 (1995) 152-155 